\title{
Representations Sociales et Triangulation: Une Application en Psychologie Sociale de la Sante
}

\author{
Thémis Apostolidis ${ }^{1}$ \\ Université de Provence
}

\begin{abstract}
RESUME - La relation du contexte social et de la santé représente un enjeu de recherche important pour les développements actuels en psychologie sociale de la santé. L'approche des représentations sociales offre un cadre d'analyse de la pensée sociale profane concernant la maladie, en rupture avec la tradition cognitiviste et individualiste dominante dans les analyses psychosociales des risques sanitaires. Sur le plan théorico-méthodologique, cette approche permet une étude contextualisée des processus sociocognitifs qui interviennent dans la construction des risques, en opérationnalisant la double nature, produit/ processus, de la représentation. Dans cette perspective, la triangulation en tant que stratégie de recherche inductive constitue une démarche privilégiée. Nous présentons une application de cette stratégie à travers une recherche sur les représentations des relations sexuelles et des risques liés au sida chez de jeunes adultes en France et en Grèce. Nous discutons la pertinence de la triangulation pour les approches "multi-niveaux" de la construction des risquesl.
\end{abstract}

Mots-clés: représentations sociales; triangulation; risques sanitaires.

\section{Social Representations and Triangulation: An Application in Social Psychology of Health}

\begin{abstract}
The relationship between social context and health represents an important research issue, which is at the centre of current developments of social psychology of the health. The social representational approach offers a frame to analyze common sense thinking about illness. This approach breaks with the cognitive and individualistic tradition that dominated the social psychological analysis of health risks. Concerning theory and methodology this approach enables a contextualized study of the socio-cognitive processes that intervene in the construction of risks through the operationalisation of the double nature -product/process- of the representation. Thus, triangulation as an inductive strategy of research constitutes a privileged approach. We present an application of this strategy through a research program concerning the representations of sexual relationships and AIDS-related risks of young adults in France and Greece. The pertinence of triangulation for the analysis of risk's construction within approaches that seek a multilevel explanation is discussed.
\end{abstract}

Key words: social representations; triangulation; sanitary risks.

\section{Representações Sociais e Triangulação: Uma Aplicação em Psicologia Social da Saúde}

\begin{abstract}
RESUMO - A relação entre o contexto social e a saúde representa um tema relevante para o desenvolvimento atual da psicologia social da saúde. A abordagem da representação social oferece uma estrutura para a análise do pensamento do senso comum sobre doença. Esta abordagem rompe com a tradição individualista que domina a análise social psicológica sobre os riscos sanitários. Quanto ao plano teórico e metodológico, esta abordagem permite um estudo contextualizado dos processos sociocognitivos que intervêm na construção dos riscos, operacionalizando a dupla natureza produto/processo da representação. Nesta perspectiva, a triangulação, bem como a estratégia de pesquisa indutiva constituem uma abordagem privilegiada. Apresenta-se uma aplicação desta estratégia por meio de uma pesquisa sobre a representação das relações sexuais e dos riscos ligados a aids entre jovens adultos na França e na Grécia. São discutidas a pertinência da triangulação para as abordagens que buscam explanações "muti-níveis" da construção dos riscos.
\end{abstract}

Palavras-chave: representações sociais; triangulação; riscos sanitários.

Parmi les développements que connaît aujourd'hui la Psychologie de la santé, les approches psychosociales centrées sur l'analyse des relations entre le contexte social et la santé

1 Endereço: Laboratoire de Psychologie Sociale, Université de Provence, 29 avenue R. Schuman, 13621 Aix-en-Provence Cédex 1, France. Email: aposto@up.univ-aix.fr représentent un champ de recherches en plein essor. Ainsi, une "psychologie sociale de la santé" se constitue depuis quelques années dans le champ de plus en plus diversifié des sciences humaines et sociales de la santé. La Psychologie Sociale de la santé peut être définie comme une pratique de psychologie sociale appliquée qui propose un ensemble des savoirs en s'appuyant sur les outils théoriques et méthodologiques à 


\section{T. Apostolidis}

la fois de la Psychologie (Psychologie Sociale, Psychologie de la santé, Psychologie Clinique) et des Sciences Sociales (Épidémiologie, Sociologie, Économie, Anthropologie). Elle est centrée sur l'étude et la résolution des problèmes de santé dans les différents contextes sociaux et culturels dans lesquels ils se manifestent (Morin \& Apostolidis, 2002). Les nouvelles options méthodologiques en Psychologie sociale de la santé visent à dépasser les oppositions classiques entre approches qualitatives et approches expérimentales, entre variables subjectives et variables objectives (Fischer \& Tarquinio, 1999). Dans ce contexte, la triangulation (Denzin, 1978), en tant que stratégie de recherche inductive (Flick, 1998), représente un enjeu à la fois théorique et méthodologique.

Afin de discuter l'intérêt de cette stratégie de recherche, cet article présente une recherche sur la construction des risques sanitaires, réalisée dans une démarche de triangulation $^{2}$. Nous commencerons par poser l'intérêt de l'approche des représentations sociales pour étudier la pensée sociale profane concernant la santé et la maladie. Nous exposerons brièvement la définition et les principes qui sous-tendent les stratégies de triangulation. Nous illustrerons une application possible de cette stratégie à partir d'un programme de recherches sur les représentations des relations intimes sexuelles et les risques liés au sida dans des populations de jeunes adultes vivant en France et en Grèce. Enfin, nous discuterons certaines questions théoriques et méthodologiques en psychologie sociale de la santé dans la perspective des représentations sociales et de la triangulation.

\section{Les Représentations Sociales: Un Regard Sur la Pensée Ordinaire de la Maladie}

Depuis la contribution princeps de Moscovici (1961), les travaux portant sur les représentations sociales se sont développés en psychologie sociale dans une pluralité des perspectives conceptuelles et méthodologiques. On peut distinguer de façon schématique trois orientations principales de travail (Jodelet, 1991): a) celle qui étudie l'incidence de la structure sociale dans l'élaboration d'une représentation (Doise, 1990); b) celle qui analyse la dynamique représentationnelle et ses caractéristiques structurales, notamment en relation avec les pratiques sociales (Abric, 1987); c) et, celle qui examine le rôle régulateur des représentations sur les interactions sociales et sur la façon dont elles interviennent en milieu réel (Jodelet, 1989). La variété et la richesse des différentes perspectives s'inscrivant dans ce champ théorique illustrent la vitalité de ce courant de recherche ayant pour dénominateur commun l'étude des aspects psychosociaux qui concourent à l'élaboration des représentations, à leurs fonctions et à leur incidence aux niveaux de la communication et de la construction de la réalité sociale.

La question de la maladie a occupé une place prépondérante dans les travaux s'inscrivant dans la théorie des représentations sociales. Différentes contributions empiriques (Joffe, 1999 Flick, 1991; Herzlich, 1969; Jodelet,

2 Nous reprenons ici l'exemple d'une recherche qui a déjà servi d'illustration dans le cadre d'un chapitre méthodologique (voir: Apostolidis, 2003)
1989; Morin, 1996) ont montré la pertinence et la fécondité de cette approche dans l'analyse et la compréhension de la dynamique psychosociale en jeu dans la construction du rapport à la maladie. Ces recherches ont permis d'illustrer comment la maladie en tant que réalité représentée renvoie à la mise en relation entre "l'ordre biologique" et "l'ordre social". Ainsi, la principale contribution de l'approche des représentations sociales de la santé et de la maladie a été de montrer que

nos représentations ne nous renseignent pas seulement sur la relation que nous entretenons avec les phénomènes corporels et notre propre état de santé, mais sur les relations qu'à travers eux, nous entretenons avec les autres, avec le monde et l'ordre social (Herzlich, 2001, p. 198).

Cette approche a mis en évidence l'assise sociale de la construction de la pensée ordinaire concernant la maladie, à travers notamment l'étude de la mobilisation des matériaux que fournit la culture (Jodelet, 2002). Les travaux sur les représentations sociales ont montré que les représentations de la maladie (signifiant) expriment sui generis une forme de rapport entre l'individuel et le social (signifié) et permettent ainsi d'accéder aux principes plus larges (appartenance, idéologie, intentionnalité) qui régissent les relations qu'entretiennent les individus avec les autres et le monde. L'apport de ce travail d'interprétation a été le déplacement vers une problématisation plus holistique des phénomènes de représentation en général, et de la maladie en particulier. C'est à travers ce type de contribution que la théorie des représentations sociales prend sa valeur heuristique de théorie paradigmatique en psychologie sociale, dont le rôle essentiel est de proposer une vision globale des relations et des comportements humains, une vision de la nature humaine (Moscovici, 1984).

Nous ne reviendrons pas ici sur une définition de la notion de représentation sociale. Cette tache ardue a déjà donné lieu à des nombreuses formalisations (Abric, 1994; Doise, 1990; Jodelet, 1984). Nous ferons trois remarques afin de préciser notre intérêt pour ce paradigme dans le cadre de la Psychologie Sociale de la santé.

Il s'agit d'un cadre théorique intégrateur (Morin, 1996) permettant de penser et d'étudier l'articulation entre dynamiques cognitives et psychologiques et situations relationnelles et sociales (correspondance entre les formes intellectuelles et les divisions sociales, Moscovici, 1961).

Il propose un modèle d'analyse de la pensée sociale qui dégage les mécanismes psychologiques et sociaux de sa production, ses opérations et ses fonctions (Jodelet, 1984) selon une conception qui pose l'individu comme sujet-acteur, socialement et culturellement situé, ayant une logique de maîtrise des situations.

Il pose le principe d'articulation entre systèmes de pensée et systèmes de comportement, des relations (orientation, légitimation) entre pratiques et représentations sociales (Abric, 1994), dans et par la considération de la correspondance entre "situations sociales" et "modalités de connaissance et d'action".

On peut définir le regard en termes des représentations sociales comme une lecture "multi niveaux". Cette lecture 
est fondée sur le questionnement "quelles régulations sociales actualisent quels fonctionnements cognitifs dans quels contextes spécifiques?" (Doise, 1990, p. 115), questionnement qui sous-tend l'incontournable articulation des différents niveaux de l'analyse psychosociale: individuel, interindividuel, positionnel, idéologique et culturel. Le regard des représentations sociales consiste à mettre en place un travail de contextualisation pour analyser la pensée sociale dans le domaine de la santé et de la maladie (Morin \& Apostolidis, 2002). Une perspective d'opérationnalisation consiste à étudier les contenus de représentation à partir des matériaux discursifs pour analyser la construction d'un "état des savoirs et des significations" et examiner "la dynamique de leur production et de leur liaison aux pratiques, aux interactions, et aux prises de position sociales" (Jodelet, 1992, p. 109). C'est dans cette perspective que nous inscrivons notre démarche. Elle s'intéresse aux catégories profanes ${ }^{3}$ de la maladie et les étudie à travers les deux processus de construction d'un objet dans la pensée de sens commun, l'objectivation et l'ancrage. En se différenciant des approches dominantes dans le champ de la cognition sociale, l'approche des représentations sociales vise le dépassement, d'une part de la rationalité en tant que modèle épistémologique pour penser l'homme, et d'autre part, de l'opposition "rationnel/irrationnel" pour étudier la pensée sociale dans le champ de la santé et de la maladie. Ainsi, cette approche rompt avec une tradition cognitiviste et individualiste en psychologie sociale, tradition dans le cadre de laquelle la perception des risques par le sujet humain est étudiée en évaluant la pensée ordinaire sur la base des standards du raisonnement rationnel et à partir d'une conception déficitaire et intra-individuelle de l'activité cognitive qui implique la notion d'erreur (Joffe, 2003).

D'un point de vue opérationnel, deux notions permettent d'analyser la pensée ordinaire de la maladie: celle de sens et celle de filtre (Apostolidis, 1994). La notion de sens, renvoie à la signification attribuée à un objet donné, à la fois au niveau individuel et au niveau social. La notion de filtre, constitué par les réseaux d'ancrage des informations et des significations concernant l'objet, renvoie au cadre interprétatif "déjà-là" à finalité pratique (maîtrise de la situation, guide pour l'action, expression). Au plan théorico-méthodologique, il s'agit de recueillir selon un cadre raisonné des matériaux discursifs pour analyser:

Les processus d'élaboration des significations par des jeux d'ancrage et d'objectivation qui construisent l'objet maladie.

Leur caractère de forme de connaissance historiquement, culturellement et socialement située.

Leur instrumentalisation dans l'interprétation et la maîtrise de la réalité sociale (étiologies, perceptions des risques), et notamment leur rôle médiateur et régulateur des interactions avec les autres (sains/malades, soignants/soignés).

3 Ces catégories de pensée sont considérées comme des catégories emics, c'est-à-dire des catégories propres à un contexte socioculturel donné. Le chercheur s'efforce de les étudier en adoptant une posture compréhensive et une "perspective de l'intérieur" (insiders perspective), et en les référant à leurs conditions historiques, sociales, et culturelles de production.

\section{Pluri-Méthodologie et Représentations Sociales: la Perspective de la Triangulation}

L'idée que l'étude des représentations sociales ne peut se satisfaire d'une seule méthode n'est pas nouvelle. Étudier, comme le suggérait Moscovici (1961), la connaissance que les individus possèdent au sujet d'un objet et la manière dont celle-ci est organisée et utilisée par les individus et les groupes, implique la perspective incontournable de la pluri-méthodologie. Rappelons que l'intérêt et la nécessité de l'approche pluri-méthodologique pour circonscrire les phénomènes représentationnels dans leur complexité sont des enjeux constamment développés et discutés (Abric, 1994; Jodelet, 1989; Hewstone, 1986). De telles approches ont permis l'avancement théorique et la production des illustrations attestant de la valeur heuristique de la théorie. Retenons de ces contributions variées que le choix et l'articulation des méthodes et des techniques de recueil posent des questions redoutables et demandent à être explicités au regard des justifications d'ordre théorique et empirique. En effet, la mise en place d'un dispositif pluri-méthodologique devrait être guidée par l'objet et le cadre théorique de l'étude. Les questions théoriques étant interdépendantes des questions méthodologiques (Di Giacomo, 1981), la diversité et l'éclectisme des approches méthodologiques qui étudient les différents aspects cognitifs et sociaux des représentations sociales attestent du développement actif de la théorie (Moscovici, 1988). L'avancement dans cette orientation méthodologique est fondamental pour produire des connaissances transposables, notamment pour les approches qui se centrent sur l'étude des représentations objectivées dans le discours social et les analysent en utilisant des méthodologies de terrain (Jodelet, 1984). Dans cette optique de traitement psychosocial de la notion de représentation sociale, nous aborderons la stratégie de triangulation.

\section{Sur la triangulation}

L'idée de triangulation repose sur un principe de validation des résultats par la combinaison de différentes méthodes visant à vérifier l'exactitude et la stabilité des observations. Initialement, la triangulation a été conçue comme une procédure pour vérifier une hypothèse, mise à l'épreuve dans des différentes opérations méthodologiques pour tester si oui ou non les résultats corroborent entre eux (Cambel \& Fiske cités par Flick, 1992). Les développements récents des approches qualitatives (Denzin, 1978; Flick, 1992) ont permis de reconsidérer la triangulation au-delà du seul critère de recoupement (usage combiné de deux ou plusieurs méthodes), en tant que stratégie alternative de recherche pour fonder une démarche épistémologique et empirique contextualisée dans les études en psychologie (Flick, 1998; Janesick, 1998; Rouan \& Pedinielli, 2001; Willig, 2001). Dans cette perspective, la stratégie de triangulation vise à conférer aux démarches qualitatives non seulement de la validité mais aussi, et surtout, de la rigueur, de l'ampleur et de la profondeur (Flick, 1992; Denzin \& Lincoln, 1998; Jodelet, 2003). Ces développements témoignent du souci de donner aux approches qualitatives de la légitimité, notamment en essayant de sensibiliser les chercheurs aux problèmes 


\section{T. Apostolidis}

d'ordre méthodologique et épistémologique que pose toute démarche de ce type (sous et surinterprétation des données, rôle et place du chercheur, potentialités et limites de chaque méthode, critères de validation -validité interne versus crédibilité ou plausibilité-).

La mise en place d'une stratégie de triangulation peut prendre différentes orientations sur les plans conceptuel et méthodologique. Denzin (1978) a distingué quatre formes de base:

1. Triangulation des données (utiliser différentes sources de données dans une étude)

2. Triangulation $d u$ chercheur (engager plusieurs chercheurs pour la collecte et l'interprétation des données)

3. Triangulation théorique (utiliser différentes théories pour interpréter les données recueillies)

4. Triangulation méthodologique (utiliser différentes méthodes et techniques pour étudier le même phénomène particulier).

Récemment, on y a ajouté une cinquième, la triangulation interdisciplinaire (Janesick, 1998): elle renvoie à la nécessaire articulation des données en psychologie à des connaissances issues de différents champs disciplinaires (Anthropologie, Histoire, Sociologie). Ces formes de triangulation peuvent être opérationnalisées de façon indépendante ou interdépendante. Leur articulation complète répond à un idéal difficilement atteignable, et dans un sens peut être infini (Becker, cité par Rouan et Pedinielli, 2001), en termes de profondeur et de finesse de l'analyse de la complexité du phénomène étudié.

Les démarches de triangulation se construisent de façon située et particulière, à partir des terrains spécifiques, en fonction de la problématique et des objectifs de la recherche, sur la base des options théoriques et épistémologiques des chercheurs. Il est difficile de définir une démarche type de triangulation et de présenter de façon formelle les étapes et les opérations à entreprendre. L'hétérogénéité et la non-superposition systématique des opérations de triangulation à partir d'un cadre prédéfini sont revendiquées dans une posture non-positiviste, comme des prérogatives de la démarche et de la capacité du "chercheur-bricoleur" (Denzin \& Lincoln, 1998) à mobiliser de façon raisonnée et éclectique des outils propres à chaque situation pour étudier la complexité des phénomènes auxquels il est confronté. En ce sens, la triangulation est avant tout une stratégie inductive de recherche (partir d'un phénomène particulier et observé sur le terrain pour le décrire et le comprendre) se donnant pour objectif général de construire un savoir pertinent et consistant sur le phénomène à partir des différentes opérations de croisement sur les plans théorique, méthodologique et/ou de production des données. On peut dire qu'elle propose une démarche analogue à celle de la Grounded Theory (Willig, 2001), définie en tant que une méthodologie de recherche qualitative qui utilise systématiquement une série de procédures pour développer une approche théorique inductive à propos d'un phénomène (Strauss \& Corbin, 1990). Selon les mêmes règles que les démarches de type grounded, elle peut être définie comme une procédure de va-et-vient problématisé et dynamique entre opérations de recueil, d'analyse et de conceptualisation. Il s'agit d'un aller-retour constant entre "terrain" et "analyse" basé sur le principe de réinjection, sous forme de questionnements et d'hypothèses, des observations de façon à concevoir et à réaliser des nouvelles opérations empiriques visant à construire une "théorie plausible" à propos d'un phénomène particulier et situé.

En psychologie sociale de la santé, le développement des procédures basées sur la triangulation, et plus particulièrement sur la complémentarité méthodologique, constitue un enjeu important. En effet, cette perspective permet (Fischer \& Tarquinio, 1999):

D'apporter du crédit (validité, réduction des biais liés à la méthode et/ou à l'outil) et de la richesse (profondeur, complexité) à l'analyse.

D'articuler "explications causalistes des comportements" et "interprétation psychosociale du sens des conduites".

\section{Triangulation et étude des contenus de représentation}

La triangulation en tant stratégie de recherche pour l'étude des représentations sociales présente un intérêt primordial. Plus particulièrement, lorsque l'on s'intéresse à l'étude des contenus spécifiques de représentation concernant la construction d'un objet du monde social. Les enjeux théoriques (statut des contenus - variant/local - et des processus -invariant/universel - ; lecture multi-niveaux) et méthodologiques (accessibilité, création des procédures ad hoc, opérationnalisation des dispositifs d'articulation multiniveaux) d'une telle perspective de recherche impliquant notamment l'étude des matériaux discursifs sont évidents. Ces enjeux sont au cœur de débats épistémiques plus globaux concernant la production des connaissances en psychologie sociale (descriptif versus explicatif, opposition en termes de validité et de légitimité entre procédures qualitatives et expérimentales). Il est clair que pour montrer l'intérêt scientifique de l'étude des contenus de représentation et pour concevoir des dispositifs variés et adaptés permettant de les analyser, l'application de la triangulation constitue une démarche potentiellement féconde et transposable. De plus, en tant que stratégie de recherche inductive, elle paraît appropriée à la spécificité épistémologique des représentations sociales, théorie paradigmatique, à visée explicative générale et à portée prédictive seulement locale (Moscovici, 2001), s'intéressant à un ensemble de phénomènes ipso facto complexes et déterminés par l'interdépendance dynamique des facteurs psychologiques, relationnels et sociaux.

Pour présenter une piste d'application illustrant l'intérêt de la stratégie de triangulation par rapport à l'avancement théorique, nous traiterons la question du rapport entre les processus et les produits de l'activité représentationnelle (Jodelet, 1994). Pour le développement de la théorie des représentations sociales, notamment dans une perspective de discussion avec les autres courants théoriques de la psychologie sociale (e.g. la cognition sociale), cette question revêt un enjeu théorique et méthodologique particulièrement intéressant, étant donné que bien des aspects restent insuffisamment développés concernant le statut de la cognition-produit. Dans cette perspective, approcher sous un angle théorique l'étude des contenus représentationnels paraît essentielle pour plusieurs raisons: du fait de la double nature de la représentation en tant que pensée constituante et pensée constituée, de la 
forme de connaissance qu'elle constitue et de son efficacité dans la construction de la réalité, de ses caractéristiques en tant produit historiquement, culturellement et socialement situé (Jodelet, 1994). Or, par exemple, bien que des travaux expérimentaux aient montré le rôle des représentations "déjàlà" en tant que systèmes d'accueil pour l'appropriation des nouvelles informations (Abric, 1987; Flament, 1984), "on a tendance à négliger le fait que l'aspect processuel se trouve en amont et en aval du produit, et seule la prise en compte des contenus permet une étude systématique des aspects processuels" (Jodelet, 1994, p. 30).

Cette piste théorique doit être davantage développée. En effet, l'articulation entre processus et contenus semble incontournable lorsque l'on s'intéresse à la façon dont les différentes constructions de la pensée sociale (construits culturels, pré-savoirs, théories implicites) affectent le traitement de l'information et la connaissance des objets sociaux (Deconchy, De Koning \& Medioni, 1993). Il s'agit là d'un enjeu théorique plus général et fondamental en Psychologie Sociale (Beauvois \& Deschamps, 1990; Deschamps \& Clémence, 1987; Farr \& Moscovici, 1984; Hewstone, 1989; Hewstone \& Augoustinos, 1998; Paicheler, 1984). Au plan théorico-méthodologique, il passe par la démonstration que l'étude des processus sociocognitifsdes (des processus par lesquels se produit un mode de connaissance indissociable des faits d'inscription et de participation sociales, c'est-à-dire des faits de culture, d'idéologie et de relations interpersonnelles et sociales) implique nécessairement la prise en compte des contenus de représentation. Car le travail sur les contenus permet de considérer les théories préexistantes des sujets et donc d'étudier leur rôle en tant que systèmes d'accueil dans le décodage et l'interprétation des informations provenant de la réalité sociale (voir par exemple, Echebarria Echabe \& Paez Rovira, 1989). Cet enjeu d'avancement théorique posé, nous présenterons maintenant l'exemple d'une démarche de triangulation réalisée à partir d'un va-et-vient problématisé entre différentes opérations de croisement sur les plans théorique, méthodologique et des données. Outre l'objectif d'illustrer cette démarche dans une optique de transférabilité, le choix de cet exemple témoigne également de notre souci de montrer comment l'étude des raisonnements en jeu dans la construction des risques sanitaires nous confronte à la dynamique sociocognitive des phénomènes de représentation.

\section{Une Ilustration: les Représentations des Relations Sexuelles Dans de Populations de Jeunes Adultes en France et en Grèce}

Précisons que la recherche présentée ici s'est inscrite dans un programme plus vaste, développé dès 1990 dans le cadre du Laboratoire de Psychologie Sociale de l'Ecole des Hautes Etudes en Sciences Sociales (EHESS) sous la direction de D. Jodelet. Les conduites de prévention et les relations établies avec autrui dans face au sida sont étudiées au regard des systèmes de représentations sociales concernant le corps, la sexualité, la contagion. Cette recherche, réalisée entre 1991 et 1994, portait sur les logiques représentationnelles donnant sens, chez les jeunes adultes, aux relations sexuelles, et leurs rapports à la construction des risques et aux conduites préventives, dans une optique comparative entre la France et la Grèce.

\section{Relations sexuelles et sida: une opportunité de contextualisation}

Nous nous sommes intéressés à l'univers de pensée relatif aux relations intimes sexuelles, dans le cadre d'une approche psychosociale de la construction du rapport à la sexualité à l'époque du sida. Les enjeux conceptuels saisis précédemment à propos des phénomènes de représentation acquièrent une importance particulière au regard de l'observation des confrontations interindividuelles dans la situation d'échange intime (Morin, 1996) avec les risques liés à cette maladie (par exemple, caractérisation et connaissance d'autrui, climats relationnels). Dans le contexte de cette maladie emblématique et d'exception, un des objectifs de santé publique a été de diffuser une connaissance appropriée à la prévention des risques d'infection et de veiller à ce qu'une information adaptée soit transmise et bien reçue afin de modifier les comportements à risques dans le domaine de la sexualité en particulier (Morin \& Apostolidis, 2002). L'histoire de la lutte préventive contre le sida est émaillée de bilans s'appuyant sur de nombreuses enquêtes de type épidémiologique focalisées sur un suivi des connaissances, des attitudes, des croyances, des comportements, en population générale. De façon transversale et récurrente, différentes observations ont mis en évidence l'existence des relations complexes entre connaissances, attitudes et comportements (niveau d'information et engagement dans des comportements à risque, connaissances acquises et connaissances fonctionnelles), et la variabilité des conduites de prévention en fonction des situations (utilisation à géométrie variable du préservatif en fonction des caractéristiques du partenaire). Prenons l'exemple de l'important dispositif de l'enquête ACSF (Analyse des Comportements Sexuels en France, Spira et al., 1993): il a permis d'observer la prééminence des stratégies "à risque" de sélection du partenaire (je fais l'amour avec des personnes dont je suis amoureux; je fais l'amour avec des personnes que je connais) et leur coexistence avec un niveau très élevé d'informations sur la prévention chez les individus.

L'analyse de ces stratégies sélectives "à risque" a posé le défi du dépassement d'une vision "épidémiologique" et individuelle des conduites sexuelles (Apostolidis, 1994). L'approche en termes de comportement sexuel a conduit à une conceptualisation individualiste et métrique de la vie sexuelle, au détriment des significations, sentiments, représentations et des dynamiques relationnelles liées au vécu sexuel. Ainsi, le comportement sexuel s'apparente à une forme de "séance individuelle" ayant plusieurs actes en option, actes déracinés du contexte subjectif, et, social et de la dynamique interpersonnelle dans lesquels ils se réalisent et puisent leur sens. Ce schéma de construction ne permet pas de considérer le contexte interactif qu'impliquent les comportements sexuels en tant que formes de relations interpersonnelles et sociales, et d'envisager la vie sexuelle dans une perspective autre que celle d'un système clos sur un sujet individuel, fût-il socialement situé. Ce qui, selon nous, constitue une lacune importante pour penser la réalité phénoménologique que donne à voir la vie sexuelle du fait 
que, dans la relation sujet/objet qu'elle implique, l'objet est tout d'abord un autre sujet (Heider, 1958; Maisonneuve, 1966). La prise en compte de cette dimension ouvre sur une autre perspective de conceptualisation: une relation intime sexuelle, comme toute relation interpersonnelle, n'est pas seulement construite par deux individus isolés l'un de l'autre, mais elle est essentiellement co-construite dans et par la communication avec ce qu'elle met en jeu au cours de leurs interactions (Strauss, 1989). Les questionnements posés par l'étude des relations intimes sexuelles se situent sui generis à l'interface de l'individuel et du collectif, en tant que processus structurés et interpersonnels.

\section{Le Dispositif de Recherche}

Sur la base d'une orientation posant la primauté du relationnel contextualisé dans la régulation des conduites sexuelles, nous avons réalisé une série de recherches (qualitatives et quasi-expérimentales) sur les systèmes de représentations donnant du sens aux relations interpersonnelles intimes afin d'étudier la place de la représentation de l'autre dans l'appréhension des risques liés au sida. Ce choix d'application du principe de triangulation méthodologique entre procédures qualitatives et quasi-expérimentales ne vise pas seulement un objectif de recoupement (apporter une validation aux analyses des matériaux qualitatifs en termes de plausibilité) mais aussi un objectif d'avancement théorique et méthodologique (montrer l'incontournable des contenus pour étudier la pensée sous son double aspect produit et processus, opérationnalisation des notions de sens et de filtre). Parallèlement, nous avons tenté d'articuler à ce type de triangulation, d'autres opérations visant à la fois un objectif de validation externe de la pertinence de la démarche (triangulation des données) ainsi qu'un objectif de construction inductive d'une théorie sur les phénomènes

Tableau 1. Les opérations de triangulation articulées.

\begin{tabular}{|c|c|c|}
\hline $\begin{array}{c}\text { Types de } \\
\text { riangulation }\end{array}$ & $\begin{array}{l}\text { Opérations de recherche } \\
\text { et de conceptualisation }\end{array}$ & Objectifs \\
\hline $\begin{array}{l}\text { Triangulation } \\
\text { méthodologique }\end{array}$ & $\begin{array}{l}\text { - Enquête qualitative par } \\
\text { entretien de recherche } \\
(\mathrm{N}=79) \text {. } \\
\text { - } 3 \text { opérations de recher- } \\
\text { che quasi-expérimentales } \\
(\mathrm{N}=1116) \text {. }\end{array}$ & $\begin{array}{l}\text { Analyse des contenus } \\
\text { et étude des aspects } \\
\text { processuels. Fournir } \\
\text { un indicateur de } \\
\text { plausibilité. }\end{array}$ \\
\hline $\begin{array}{l}\text { Triangulation des } \\
\text { données }\end{array}$ & $\begin{array}{l}\text { Mise en perspective } \\
\text { comparée avec les don- } \\
\text { nées épidémiologiques. } \\
\text { Source de comparaison: } \\
\text { l'enquête l'ACSF (1993). } \\
\text { Enquête épidémiologique } \\
\text { sur les comportements } \\
\text { sexuels, réalisée à } \\
\text { partir d'un échantillon } \\
\text { représentatif de la popu- } \\
\text { lation vivant en France. }\end{array}$ & $\begin{array}{l}\text { Recoupement avec } \\
\text { d'autres sources de } \\
\text { données. Fournir un } \\
\text { indicateur de valida- } \\
\text { tion externe de la } \\
\text { démarche (pertinence, } \\
\text { intérêt) }\end{array}$ \\
\hline $\begin{array}{l}\text { Triangulation } \\
\text { interdisciplinaire }\end{array}$ & $\begin{array}{l}\text { Problématisation et con- } \\
\text { frontation des analyses au } \\
\text { regard des contributions } \\
\text { sociologiques, anthro- } \\
\text { pologiques et historiques } \\
\text { sur la sexualité et la } \\
\text { maladie. }\end{array}$ & $\begin{array}{l}\text { Articuler différents } \\
\text { niveaux d'analyse, } \\
\text { notamment des } \\
\text { explications de type } \\
4 \text { (facteurs sociocul- } \\
\text { turels) }\end{array}$ \\
\hline
\end{tabular}

Tableau 2. Protocole utilisé pour la réalisation des entretiens sur la sexualité (Apostolidis, 2001).

\begin{tabular}{|c|c|}
\hline Composition du protocole & $\begin{array}{c}\text { Exemples de relances verbales } \\
\text { utilisées }\end{array}$ \\
\hline Consigne de départ & $\begin{array}{l}\text { “J'aimerais que l'on parle de } \\
\text { la sexualité. Que pouvez-vous } \\
\text { m'en dire? Qu'évoque pour } \\
\text { vous la sexualité?” }\end{array}$ \\
\hline $\begin{array}{l}\text { Les thèmes du guide: } \\
\text { La place de la sexualité dans la } \\
\text { vie de la personne (accomplisse- } \\
\text { ment personnel, relations avec les } \\
\text { autres) }\end{array}$ & $\begin{array}{l}\text { "À votre avis, quelle place occupe } \\
\text { la sexualité dans la vie d'une } \\
\text { personne?" } \\
\text { "Pensez-vous que la sexualité } \\
\text { contribue à l'accomplissement } \\
\text { d'une personne? De quelle } \\
\text { manière?" }\end{array}$ \\
\hline La notion du besoin sexuel. & $\begin{array}{l}\text { "Selon-vous, peut-on l'associer à } \\
\text { un besoin?" }\end{array}$ \\
\hline Le sens donné au plaisir. & $\begin{array}{l}\text { "Que pouvez-vous me dire par } \\
\text { rapport au plaisir?" }\end{array}$ \\
\hline Le lien entre sexualité et amour. & $\begin{array}{l}\text { “Qu'évoque pour vous l'amour } \\
\text { par rapport à la sexualité? Que } \\
\text { signifie l'amour pour vous? C'est } \\
\text { quoi une relation amoureuse? }\end{array}$ \\
\hline La fidélité & $\begin{array}{l}\text { "La fidélité, c’est quoi pour } \\
\text { vous?" }\end{array}$ \\
\hline $\begin{array}{l}\text { Le sida, les risques associés, com- } \\
\text { portements à cet égard, étiologies, } \\
\text { attitudes vis-à-vis du préservatif } \\
\text { et de la prévention. }\end{array}$ & $\begin{array}{l}\text { "Et par rapport au sida, que pou- } \\
\text { vez-vous m'en dire? À quoi cette } \\
\text { maladie vous fait-elle penser?" } \\
\text { "Comment s'en prémunit-on?", } \\
\text { "Qu'évoque pour vous le préser- } \\
\text { vatif?", "Que pensez-vous des } \\
\text { campagnes de prévention?", "Et } \\
\text { par rapport à vous-même? Est-ce } \\
\text { que le sida vous préoccupe? Que } \\
\text { faites-vous?" }\end{array}$ \\
\hline Identification des interviewés & $\begin{array}{l}\text { Age, sexe, niveau scolaire, profes- } \\
\text { sion, relation stable. }\end{array}$ \\
\hline
\end{tabular}

permettant d'envisager les conditions sociales et culturelles de leur production (triangulation interdisciplinaire). Bien évidemment, nous ne pouvons prétendre discuter ici ni tous les détails des opérations de recherche et de conceptualisation réalisées, ni l'ensemble des questions qu'elles soulèvent tant par leurs limites inhérentes (recueil et analyse des matériaux, conditions d'application) que par les nouveaux questionnements théoriques qu'elles ont permis d'envisager. Notre objectif consiste à présenter une esquisse de triangulation raisonnée posant un regard "multi-niveaux" pour atteindre certains aspects de la dynamique et de la complexité des phénomènes de représentation auxquels nous confronte l'analyse de l'univers de pensée relatif aux relations sexuelles. Nous pouvons présenter la démarche et les objectifs de différentes opérations réalisées de façon schématique (cf. Tableau 1). Nous exposerons en trois étapes des opérations de va-et-vient pour le recueil et l'analyse.

\section{$1^{\mathrm{e}}$ étape: Les entretiens de recherche}

\section{Echantillon et protocole}

Nous avons réalisé 79 entretiens de recherche sur le thème de la sexualité en France $(N=38)$ et en Grèce $(N=41)$, 
Encadré 1. Les quatre étapes de l'IPA (Smith, 1995).

L'Analyse Phénoménologique Interprétative (IPA) est une pratique d'analyse appropriée pour les entretiens semi-directifs. On distingue quatre phases:

- $1^{\mathrm{e}}$ phase: Immersion et familiarisation avec les données du corpus (lecture et relecture de l'ensemble des entretiens, annotations) visant à réaliser un codage exploratoire à partir des premiers éléments de questionnement et d'interprétation.

- $2^{\mathrm{e}}$ phase: Inventaire et identification systématiques des thèmes émergeants du corpus analysé et labellisation conceptuelle de ces thèmes pour construire une grille d'analyse.

- $3^{\mathrm{e}}$ phase: Application de la grille construite pour mettre à jour les relations entre les différents thèmes (hiérarchisation, univers commun de significations) illustrées notamment par des extraits de données brutes. Élaboration des clusters (catégories thématiques) à partir des connexions entre les différents thèmes et sous-thèmes.

- $4^{e}$ phase: Production pour chaque entretien de tableaux-résumé des catégories les plus signifiantes rendant compte de l'expérience singulière des interviewés. Le choix d'inclure telle ou telle catégorie est fonction des matériaux, de la problématique et des objectifs de la recherche. Ces tableaux regroupent pour chaque entretien quatre types d'informations: les sous-thèmes labellisés de chaque catégorie, les éléments conceptuels associés, les extraits in vivo et leurs coordonnées au sein de l'entretien (numéro de page, numéro de ligne).

Ces différentes phases conduisant à la réalisation des tableaux-résumé pour chaque entretien vont servir pour construire des grilles d'analyse pour analyser l'ensemble du corpus.

Tableau 3. Exemple d'une fiche individuelle d'analyse issue de codage axial (entretien par entretien).

\begin{tabular}{|c|c|c|c|}
\hline Thèmes & Éléments d'analyse & Extraits & $\begin{array}{l}\text { Indicateur Page/ } \\
\text { Séquence }\end{array}$ \\
\hline \multirow{4}{*}{$\begin{array}{l}\text { Liens entre sexualité et senti- } \\
\text { ments amoureux }\end{array}$} & \multirow{2}{*}{$\begin{array}{l}\text { Évocation spontanée } \\
\text { Jugement évaluatif } \\
\text { Ajout de sens: bestial } \\
\text { Expression/préférence }\end{array}$} & $\begin{array}{l}\text { "La sexualité, ça vient après des relations purement } \\
\text { sentimentales... sinon c'est plutôt bestial, je ne suis pas } \\
\text { tellement pour" }\end{array}$ & $1 / 1$ \\
\hline & & $\begin{array}{l}\text { "Il faut qu'il y ait des sentiments, c'est un avis person- } \\
\text { nel, moi je me sens mieux" }\end{array}$ & $1 / 2$ \\
\hline & $\begin{array}{l}\text { Différence entre deux formes } \\
\text { de relation: } \\
\text { Égoïsme vs partage }\end{array}$ & $\begin{array}{l}\text { "c'est une relation bestiale, on cherche ni plus ni moins } \\
\text { à soutirer du plaisir à la personne alors que la relation } \\
\text { sentimentale, on essaie de s'apporter quelque chose } \\
\text { mutuellement, c'est un rapport d'égalité" }\end{array}$ & $2 / 3$ \\
\hline & Connaissance d'autrui & $\begin{array}{l}\text { "c'est travers les relations sentimentales et non sex- } \\
\text { uelles qu'on arrive à connaître la personne" }\end{array}$ & $11 / 11$ \\
\hline
\end{tabular}

auprès de jeunes adultes $(18-26$ ans), hommes $(N=41)$ et femmes $(N=38)$. La campagne des entretiens s'est déroulée à Paris et à Athènes. L'objectif des enquêtes qualitatives n'est pas la construction d'un échantillon représentatif. Le recrutement (technique de boule-de-neige, mise en relation par des contacts institutionnels) a été opéré de façon progressive et ciblée afin de diversifier les profils individuels selon certains critères (âge, sexe, niveau scolaire, insertion professionnelle, modes de vie). Nous avions ainsi cherché à obtenir une "hétérogénéité qualitative" de l'échantillon à partir de différentes situations jeunes (étudiants, jeunes insérés professionnellement, sans emploi, ...).

Les entretiens ont été conduits selon la technique de la semi-directivité. La réalisation des entretiens sur le thème de la sexualité (voir protocole Tableau 2) avait deux objectifs: a) la non-imposition de la problématique "sida" aux interviewés (cf. Demazière \& Dubar, 1997); b) l'opérationnalisation d'un protocole permettant d'étudier la construction des objets sociaux complexes, comme le sida, en explorant des univers de pensée plus larges, comme celui de la sexualité (cf. Jodelet, 1984).

\section{Sur l'analyse thématique des entretiens}

Les entretiens, d'une durée d'une heure en moyenne, ont été intégralement retranscrits à l'aide d'un logiciel de traitement de texte. Nous avons réalisé une analyse thématique systématique aux niveaux vertical (sur chaque entretien) et horizontal (sur l'ensemble des entretiens). L'analyse thématique des matériaux discursifs par entretien de recherche repose sur le repérage des contenus se référant à des "thèmes", c'est-à-dire à des unités de signification isolables et analysables de façon transversale (Bardin, 1977), selon différents critères quantitatifs (fréquence ou typicité du thème par rapport à une sous-population de l'échantillon) et qualitatifs (saillance du thème par rapport à la problématique). L'Analyse Phénoménologique Interprétative (Smith, 1995; Willig, 2001; cf. Encadré 1) offre un cadre de travail pour réaliser une analyse thématique visant à dégager "des analogies de structure et de contenu" (Michelat \& Simon, 1977), à partir d'un corpus très composite où "aucun entretien n'est identique à un autre".

Après la lecture de tous les entretiens (phase 1), nous avons effectué un inventaire thématique (thèmes spontanés 


\section{T. Apostolidis}

du guide; phase 2) pour construire une grille de codage axial (codage à partir d'un système préliminaire des codes; cf. Strauss \& Corbin, 1990). Sur la base de cette grille, nous avons mis en place un double procédé d'analyse:

Une recherche thématique au niveau de chaque entretien (phase 3) à plusieurs niveaux (catégories thématiques utilisées: aspects psychosociaux de la sexualité; sexualité, corps et besoin; sexualité et attraction interpersonnelle (formes de relation, critères de classement); liens entre sexualité et sentiments amoureux; notion de désir et de plaisir; sida, risques associés et conduites évoquées). Nous avons ainsi construit des grilles interindividuelles d'analyse pour l'ensemble du corpus.

Une analyse de la singularité de chaque entretien (enchaînement, connexions et importance de chaque thème, posture d'énonciation). Nous avons utilisé trois indicateurs pour chacun des thèmes de la grille (ordre chronologique d'apparition, succession et modalisation, fréquence intra). Chaque fois que nous rencontrions un thème, nous lui avons associé un code numérique correspondant à l'unité de découpage où il se trouve (numéro de page et numéro de séquence de locution). Ces informations ont été recueillies et systématisées à partir du dépouillement de chaque entretien sur la base de la grille. Cette analyse a abouti à la création des fiches individuelles (phase 4; cf. Tableau 3).

Rappelons que toutes ces opérations de codage et d'analyse des productions discursives complexes, hétérogènes, irréductibles, et obéissant à des règles de production et d'énonciation variées, aussi minutieuses et rigoureuses qu'elles soient, posent de fait plusieurs problèmes: objectivité et pertinence des catégories utilisées pour le codage axial, sur ou sous-interprétation, ... L'objectivité et la standardisation représentent des objectifs qui ne sont jamais véritablement atteignables dans l'étude de ce type de matériaux, voir même qui peuvent constituer des freins pour la profondeur et la créativité de l'analyse. C'est alors que les différents types de triangulation (méthodologique, de données) offrent des alternatives de validation par déplacement raisonné des critères de validité (précision de la mesure versus recoupement, validité interne versus plausibilité) pour décider de l'orientation que peuvent prendre l'analyse et l'interprétation.

\section{Principaux constats}

De quoi parle-t-on quand on parle de sexualité? L'analyse des thèmes évoqués spontanément (sans induction de l'interviewer $)^{4}$ nous a conduit à considérer l'univers de l'amour et des sentiments amoureux en tant que lieu de mise en discours et de construction du rapport au sexuel. En filigrane des multiples facettes d'appréhension et de formation de la sexualité en tant qu'objet représenté, nous avons observé que la construction du rapport à l'autre et le contexte interpersonnel dans lequel l'expérience du sexuel est envisagée impliquent de façon spontanée, transversale et récurrente dans les discours, une "thématique amoureuse".

4 Pour réaliser cet inventaire, nous avons travaillé à partir de l'unité, chronologique et textuelle, des trois premières pages dactylographiées de chaque entretien.
“Tout de suite là, sans réfléchir ... l'amour, le couple, enfin l'amour que tu as pour quelqu'un, en fait ça en découle ... s'il y a sexualité, il doit y avoir amour" $(\mathrm{F} 2, \mathrm{~F}, 1)^{5}$.

"Pour moi, c'est l'expression d'un amour, d'une tendresse, l'aboutissement d'une relation entre deux personnes de sexe opposé, je ne crois pas qu'il s'agit seulement d'un acte de plaisir" (G22, H, 1).

De façon quasi-systématique, les femmes, tant en Grèce qu'en France, n'envisagent la sexualité qu' associée à l'amour ou à des différents types de lien affectif. Chez les hommes, ce mode de représentation introduit une autre dialectique d'appréhension: "sexualité" et "amour" sont vus comme deux façons potentielles d'envisager le rapport au sexuel. En dépit de difficultés d'analyse (hétérogénéité des expressions singulières, différences hommes/femmes, conceptions spécifiques en France et en Grèce, polysémie de la référence à l'amour, par exemple), l'examen de la thématique a permis d'identifier un mode de construction distinctive entre différents types de relations sexuelles: quand il y a de l'amour et quand il n'y a pas, avec ou sans sentiments amoureux. Ce mode est médiatisé par des raisonnements qui sous-tendent des modes de perception et d'appréciation, fondés sur la distinction entre ces deux types de relation sexuelle.

"Ce genre de rencontres qui se font lors d'une soirée, tout de suite ça se termine au lit et le lendemain plus personne n'en entend parler quoi, je veux dire que c'est le genre de choses qui arrivent, c'est un peu banal quoi, et c'est ça qui tue le reste, qui tue l'amour, qui tue les vrais sentiments, qui tue plein de choses" (F38, F, 3).

Ce mode de représentation joue un rôle médiateur dans le rapport à l'autre, à travers notamment l'aspect générateur des jeux de significations pour qualifier les climats relationnels dans le contexte d'une relation sexuelle. Ce rôle s'est révélé particulièrement signifiant pour saisir l'appréhension des risques liés au sida et les significations associées aux conduites sexuelles (thématique abordée spontanément par 29/38 interviewés en France et 18/41 en Grèce).

"Dans des relations qui ne durent pas longtemps, où l'on n'est pas sûr du partenaire, le préservatif s'impose, c'est une obligation ... la différence avec le préservatif, avec la nonutilisation du préservatif, on fait l'amour et avec l'utilisation du préservatif, on baise (...) bon, je l'utilise à tous les coups, bon, si cette relation dure, on voit après" (F17, H, 7).

De plus, l'étude des discours concernant les confrontations interindividuelles intimes avec les risques associés au sida nous a permis d'observer le rôle prépondérant de ce mode de représentation dans l'élaboration des différentes "stratégies préventives" relatées par les interviewés. Variant tant du point de vue intra-individuel, que du point de vue interindividuel, nombre de ces stratégies renvoient à des

5 Pour situer les extraits d'entretiens: un code indiquant le pays ( $\mathrm{F}=$ France, $\mathrm{G}=$ Grèce, le numéro de l'entretien, le sexe de l'interviewé $(\mathrm{H} / \mathrm{F})$ et l'indication d'apparition $\left(\mathrm{N}^{\circ}\right.$ page). Les extracts d'entretiens sont choisis en raison de leur typicité 
modes de prévention bricolés et variables, faits d'écarts entre connaissances et pratiques, entre décisions et actions, à partir des conduites fluctuantes en fonction des partenaires, des espaces de rencontre (familiers versus impersonnels), des climats relationnels (confiance en tant que "bien" interpersonnel) et des aléas de situations (désirs et sentiments éprouvés, excitation et laisser-aller).

“je crois qu'on ne peut pas ignorer le sida à l'heure actuelle, on est conscient et puis ça c'est une actualité, on en parle tellement souvent, c'est mon point de ligne, ne pas foncer dans une relation sexuelle comme ça à l'heure actuelle sans savoir avec qui on va, qui vous a précédé quand même" (F1, H)

"moi je pense qu'il faut connaître la personne, c'est pas $\mathrm{du}$ jour au lendemain un rapport sexuel, ça c'est pas beau, c'est moche... parce que je sais pas, ils ne se connaissent même pas, et si ça se trouve, il y en a un qui est malade, il y en a un qui est... c'est vrai, ils ne se connaissent pas, comme ça c'est... ça ne se fait pas" (F26, F)

"il faut aussi sélectionner celles avec qui tu vas, d'accord, si une fille te dit qu'elle a le sida tu ne vas pas avec, mais qui va te le dire, et si elle ne sait pas, pour ça je pense que quand tu fais l'amour avec une fille que tu ne connais pas, tu prends toujours un risque" $(\mathrm{G} 10, \mathrm{H})$

Les discours analysés permettent d'identifier des formes de bricolage d'une "autre prévention", par des associations composites entre des savoirs véhiculés par les campagnes de prévention (usage du préservatif) et des savoirs ancrés dans des conceptions préexistantes au sein de la structure sociale concernant la sexualité (licence sexuelle et maladie, lieux de vénalité, groupes à risque, sexualité avec ou sans sentiments amoureux). Les différentes stratégies (tri sélectif des partenaires, usage du préservatif selon le cas, attente, présomption, déni) ne s'excluent pas mutuellement, mais coexistent souvent au sein d'une même trajectoire individuelle en fonction des contextes relationnels (utilisation à géométrie variable du préservatif en fonction des caractéristiques du partenaire et de la relation).

Sur la base de ces premiers constats, avant tout illustratifs d'un premier stade dans l'analyse des matériaux, nous avons cherché à dégager les caractéristiques typiques (qualitativement et quantitativement) pour faire un inventaire de différentes dimensions qui fondent la distinction entre relations sexuelles "avec" ou "sans" sentiments amoureux (par exemple: "faire l'amour" versus "baiser"; communication versus non-communication; difficile versus facile; impliquante versus pas impliquante; profonde versus superficielle; extraordinaire versus banal; valorisante versus dévalorisante; contact versus fusion; plus ou moins à risque par rapport au sida). À partir de l'étude des raisonnements étayant ces dimensions, nous avons construit une typologie "qualitative" (privilégiant la richesse et l'exhaustivité des informations et non seulement leur fréquence) de cette distinction pour disposer d'une "catégorie d'analyse" inductive permettant d'avancer au niveau de l'interprétation (cf. Tableau 4).

Afin de statuer sur la crédibilité et l'intérêt de cette piste d'analyse en tant qu'hypothèse de travail (contenu et organisation de la représentation), nous avons réalisé une série d'opérations quasi-expérimentales visant à examiner sa pertinence.

\section{$2^{\mathrm{e}}$ étape: Triangulation méthodologique par des opérationnalisations quasi-expérimentales}

Pour tester cette hypothèse de travail, nous nous sommes centré sur l'analyse des modes de connaissance d'autrui dans le contexte d'une relation sexuelle à partir de l'étayage de la distinction avec ou sans sentiments amoureux dans les discours. La production des significations que génère ce mode de représentation a permis d'identifier un certain nombre de paramètres (avec qui?; où? comment?; dans quel but?) en fonction desquels se construit la valence sentimentale perçue d'une relation sexuelle. Ces paramètres (typologie de l'autre, conditions de la rencontre) façonnent la co-construction des climats relationnels sur la base d'un système des codes et des règles qui régissent la communication et les interactions dans le domaine des échanges intimes. Différents raisonnements analysés illustrent comment la construction des relations sexuelles avec sentiments induit un climat de rencontre entre deux imaginaires (Maisonneuve, 1966), dans laquelle le partenaire est "connu" sur une base de reconnaissance et/ou de

Tableau 4. Typologie qualitative de la distinction entre les deux dégagée par l'analyse du corpus des entretiens (Apostolidis, 2001).

\begin{tabular}{ll}
\hline \multicolumn{1}{c}{ Relation sans sentiments amoureux } & Relation avec sentiments amoureux \\
\hline Vécu éphémère & Vécu à vocation de durer \\
Uniquement pour le plaisir & Exprimer ou construire quelque chose \\
Indifférence, autrui interchangeable & Attachement, autrui choisi et privilégié \\
Plus à risque & Moins à risque \\
L'autre est un "inconnu" & Connaissance et reconnaissance de l'autre \\
Par besoin et/ou désir sexuel. & Désir de l'autre, y compris sexuel \\
Peu ou pas d'implication émotionnelle & Forte implication émotionnelle \\
Relation superficielle, égoïsme & Union, fusion, profondeur, don de soi \\
Pas de communication et de réciprocité, méfiance & Respect, réciprocité, communication, partage, confiance, sincérité. \\
Immaturité, individualisme, frustration & Maturité, réalisation de soi, équilibre \\
Solution de facilité & Choix, effort et volonté \\
\hline
\end{tabular}




\section{T. Apostolidis}

Tableau 5. Présentation de deux scénarios opérationnalisant la variable "délai".

\begin{tabular}{ll}
\hline \multicolumn{1}{c}{ Rapport immédiat (sans délai) } & \multicolumn{1}{c}{ Rapport différé (avec délai) } \\
\hline Paul est en vacances pour quelque temps dans un village près de la mer. & Paul est en vacances pour quelque temps dans un village près de la mer. \\
Marie passe ses vacances d'été dans les environs. Un matin Paul et Marie & Marie passe ses vacances d'été dans les environs. Un matin Paul et Marie \\
se rencontrent au moment du petit-déjeuner dans un café à côté de la & se rencontrent au moment du petit-déjeuner dans un café à côté de la \\
plage. Ils font connaissance et après avoir bavardé un peu, ils décident & plage. Ils font connaissance et après avoir bavardé un peu, ils décident \\
d'aller se baigner ensemble. Ils passent le reste de la journée l'un avec & d'aller se baigner ensemble. Ils passent le reste de la journée l'un avec \\
l'autre à la plage. Le soir, ils vont manger dans un petit restaurant. Ils & l'autre à la plage. Le soir, ils vont manger dans un petit restaurant. Le \\
passent la soirée dans les bras l'un de l'autre. Ils finissent la nuit en faisant & $\begin{array}{l}\text { lendemain, ils se rencontrent à nouveau et ils ne se quittent plus. Quelques } \\
\text { l'amours après, ils passent la soirée dans les bras l'un de l'autre. Ils finissent }\end{array}$ \\
\hline
\end{tabular}

présupposition. Parmi les paramètres en jeu dans la construction de ce climat, nous avons identifié le rôle modalisant du délai séparant le début d'une rencontre à l'aboutissement à une relation sexuelle, les relations sans délai étant associées à l'univers des relations sans sentiments amoureux (superficielles, éphémères, vénales, égoïstes, à risque). Ce principe d'attente s'est révélé également signifiant pour saisir les ressorts psychosociaux (sens, intention, fonction) de l'adoption des stratégies préventives face au sida (sélection ou évitement des partenaires, confiance, utilisation variable du préservatif en fonction de l'évolution de la rencontre).

Sur la base de ces constats, nous avons décidé de retenir cette dimension de délai (règle de conduite: rapport avec ou sans délai) pour tenter de construire expérimentalement une opérationnalisation de la distinction des relations sexuelles avec ou sans sentiments amoureux. Nous avons réalisé trois opérations quasi-expérimentales à partir de ce dispositif: une première sur la perception d'une relation sexuelle dans des conditions avec ou sans délai $(N=482)$, deux autres sur les explications d'une contamination par le VIH en fonction de ces mêmes types de relations $(N=634)$. Rappelons que l'objectif théorique visé par ces opérationnalisations a été de produire des illustrations quasi-expérimentales permettant de montrer la pertinence de l'approche des représentations sociales (contenu, processus) pour étudier les processus sociocognitifs.

\section{Population et procédure}

Nous présentons ici les principaux résultats de la première recherche réalisée auprès d'une population d'étudiants, en France et en Grèce, dont l'objectif était de mettre en évidence expérimentalement l'impact de ce facteur sur les représentations d'une relation sexuelle. 482 sujets, étudiants et étudiantes de 18 à 25 ans, 240 en France (Paris) et 242 en Grèce (Athènes), répartis dans chaque pays en 16 groupes expérimentaux, ont pris connaissance d'un scénario de rencontre aboutissant à une relation sexuelle avec ou sans délai, avec une partenaire socialement marquée (endo ou exo-groupe). Pour la moitié des groupes, le rapport sexuel était signalé comme "protégé" (avec préservatif). Afin d'illustrer la forme qu'ont prise ces scénarios, nous présentons l'exemple (cf. Tableau 5) de l'opérationnalisation de la variable "rapport". L'opérationnalisation de la variable "partenaire" (PA) a été réalisée en modifiant le prénom de l'acteur femme: Marie, pour la condition "endo" et, Ingrid, pour la condition partenaire "exo", en y ajoutant la mention "jeune étrangère" pour accentuer la différenciation. Celle de la variable "préservatif" (P) a été introduite avec la mention "ils ont utilisé des préservatifs", à la fin du scénario dans les conditions correspondantes..

Après avoir pris connaissance du scénario, les sujets devaient faire part de leurs impressions à propos de deux protagonistes et décrire la relation évoquée. Le choix de ces items, issus de l'analyse des entretiens, répondait à deux objectifs: d'une part étudier la place de la valence sentimentale dans l'organisation du champ de la représentation d'une relation sexuelle, de l'autre, cerner les psychologies naïves mobilisées pour décrire les acteurs engagés dans ces relations. Nous ne présenterons ici que les résultats concernant la représentation de la relation induite par les scénarios (neuf items utilisés: relation qui vous plaît, sans avenir, sexuelle, sentimentale, pour le plaisir, pour construire quelque chose, de communication, superficielle, à risque par rapport au sida).

\section{Hypothèses}

Nous avons posé trois hypothèsesn principales:

L'organisation du champ de la représentation d'une relation sexuelle implique une prise de position sur la dimension de la valence sentimentale.

- La variable "rapport" induit une variation de la valence sentimentale perçue.

La perception du risque VIH varie en fonction de la valence sentimentale attribuée à la relation.

\section{Principaux Résultats}

Nous avons soumis les réponses concernant la description de la relation à une analyse en composantes principales (ACP) de façon à dégager les dimensions organisant ce champ représentationnel (cf. Doise, Clemence \& Lorenzi-Cioldi, 1992). Globalement, on observe (cf. Tableau 6, moyennes de différents items) que les relations induites par les scénarios sont perçues comme relevant plutôt d'une sexualité éphémère et hédoniste et moins de l'univers des sentiments amoureux.

Les trois analyses réalisées (une séparément pour chaque pays et une pour l'ensemble; cf. Tableau 6) ont permis de retenir deux facteurs. Elles montrent que de façon analogue, en France et en Grèce, le champ représentationnel s'organise autour de deux facteurs expliquant plus de $59 \%$ de la variance. Le premier saturé par six items oppose une vision 
Tableau 6. Présentation des 3 ACP (une sur l'ensemble, une pour chacun des deux pays), solution Varimax, sur les neuf traits décrivant la relation (Echelle utilisée: $1=$ pas du tout d'accord / $10=$ tout à fait d'accord).

\begin{tabular}{|c|c|c|c|c|c|c|c|c|c|c|c|c|}
\hline & \multicolumn{4}{|c|}{ Ensemble $(\mathrm{KMO}=.838) \mathrm{N}=482$} & \multicolumn{4}{|c|}{ Grèce $(\mathrm{KMO}=.842) \mathrm{N}=242$} & \multicolumn{4}{|c|}{ France $(\mathrm{KMO}=.836) \mathrm{N}=240$} \\
\hline & F1 & F2 & & & F1 & F2 & & & F1 & F2 & & \\
\hline$\%$ variance & 36.98 & 22.29 & & & 31.96 & 27.40 & & & 39.65 & 20.02 & & \\
\hline Traits & & & $\mathbf{m}$ & SD & & & $\mathbf{m}$ & SD & & & m & SD \\
\hline Qui plait & .752 & & 6.1 & 2.9 & .829 & & 5.9 & 2.9 & .725 & & 6 & 2.9 \\
\hline Superficielle & -.726 & .390 & 6.6 & 2.7 & -.637 & .513 & 6.4 & 2.6 & -.768 & .315 & 5.6 & 2.7 \\
\hline Construire & .713 & -.310 & 3.6 & 2.3 & .560 & -.496 & 3.2 & 2.1 & .759 & & 4 & 2.4 \\
\hline Communication & .708 & & 6.1 & 2.5 & .667 & & 5.5 & 2.5 & .696 & & 6.6 & 2.4 \\
\hline Sentimentale & .700 & & 5.4 & 2.5 & .633 & & 4.9 & 2.5 & .703 & & 6 & 2.4 \\
\hline Sans avenir & -.590 & .399 & 57 & 2.5 & -.486 & .402 & 5.7 & 2.5 & -.705 & .378 & 5.7 & 2.5 \\
\hline Plaisir & & .815 & 7.8 & 2.3 & & .773 & 7.4 & 2.5 & & .789 & 8.3 & 1.9 \\
\hline Sexuelle & & & 8.1 & & & .755 & 8 & 2 & & .764 & 8.3 & 2 \\
\hline
\end{tabular}

Note: Nous présentons les saturations supérieures à .30.

valorisante de la relation (qui plaît, sentimentale, de communication, pour construire) à une vision dévalorisante (superficielle, sans avenir). Le deuxième saturé principalement par deux items est associé au caractère hédoniste de la relation (caractère sexuel, recherche de plaisir). Nous avons posé un premier constat: la représentation d'une relation sexuelle est organisée autour de deux principes: le premier concerne la valence sentimentale, le deuxième le caractère hédoniste.

Nous avons ensuite cherché à examiner les effets de la variable expérimentale "rapport" sur la perception de la relation induite par le scénario à partir de ces deux dimensions organisatrices due champ de la représentation. Pour ce faire, nous avons construit deux indicateurs à partir des résultats de l'analyse factorielle globale: l'indicateur "dimension sentimentale" et l'indicateur "dimension hédoniste" en agrégeant les items à partir de leurs saturations sur les deux facteurs dégagés par l'analyse. Pour la dimension sentimentale, nous avons agrégé six items (qui vous plaît, de communication, sentimentale, pour construire quelque chose, sans avenir -inversé-, superficielle-inversé-; valeurs des alpha: Globale $=.815$, Grèce $=.798$, France $=.826$; division de la somme par 6). Pour la dimension "hédoniste" 2 items (sexuelle, pour le plaisir; valeurs des alpha: Globale $=.615$, Grèce $=$
.677 , France $=.517$; division de la somme par 2). L'étude de corrélations entre ces deux indicateurs suggère que les deux dimensions sont opposées, et cette opposition s'avère plus marquée en Grèce qu'en France (Global, $r=$-.340; Grèce, $r=-.530$; France, $r=-.200 ; p<.01)$. Pour tester les effets des variables indépendantes, invoquées et manipulées, nous avons soumis chacun de ces indicateurs à une ANOVA selon un plan factoriel à cinq facteurs: Pays $2 *$ Délai $2 *$ Sexe $2 *$ Partenaire $2 *$ Présevatif 2 . Les résultats présentés dans le Tableau 7 montrent qu'indépendamment des variables expérimentales, les relations induites par les scénarios sont davantage valorisées (perçues comme plus sentimentales) d'une part, par les sujets français, de l'autre, par les hommes. Conformément à notre deuxième hypothèse, nous avons observé que le facteur "rapport" faisait varier significativement la perception de la relation sur les deux indicateurs: dans la condition "rapport immédiat", la relation est perçue comme moins sentimentale et plus hédoniste. Les résultats de l'interaction entre les facteurs "rapport" et "partenaire" suggèrent qu'il s'agit davantage d'une norme de jugement endo-groupe (la caractérisation sentimentale de la relation varie uniquement lorsque la partenaire fait partie de l'endo-groupe), contexte relationnel davantage valorisé par les sujets (effet simple du facteur "partenaire").

Tableau 7. ANOVA sur les deux indicateurs (présentations des résultats significatifs pour les effets simples et les interactions de premier niveau).

\begin{tabular}{|c|c|c|}
\hline & Dimension "sentimentale" $(m=5.2$ & Dimension "hédoniste" $(m=8)$ \\
\hline Pays (A) & $m-\mathrm{GR}=4.9<m-\mathrm{FR}=5.4 * * *$ & $m-\mathrm{GR}=7.7<m-\mathrm{FR}=8.3 * * *$ \\
\hline Rapport (B) & $m-\mathrm{IMM}=4.9<m-\mathrm{DIF}=5.5 * *$ & $m-\mathrm{IMM}=8.3>m-\mathrm{DIF}=7.7 * * *$ \\
\hline Sexe $(C)$ & $m-\mathrm{H}=5.5>m-\mathrm{F}=4.9 * *$ & ns \\
\hline Partenaire (D) & $m-\mathrm{EXO}=5<m-\mathrm{ENDO}=5.4 *$ & ns \\
\hline Préservatif (E) & ns & $m$-OUI $=7.8<m-\mathrm{NON}=8.1 *$ \\
\hline $\mathbf{B} * \mathbf{D}$ & $\begin{array}{c}m \text {-IM } M-\mathrm{EXO}=m \text {-DIF-EXO } \\
\mathrm{m}-I M M-E N D O<\mathrm{m}-D I F-E N D O *\end{array}$ & ns \\
\hline
\end{tabular}

$* p<.05 ; * * p<.01 ; * * * p<.001$ 


\section{T. Apostolidis}

L'ensemble de ces résultats semble confirmer l'importance de deux dimensions (sentimentale, hédoniste) en tant que dimensions organisant le champ représentationnel de la relation induite par le scénario. Ils montrent en outre que les prises de positions sur ces deux dimensions dépendent étroitement des règles (conduite à adopter) et des facteurs socialement marqués (typologie de l'autre). Ils vérifient nos hypothèses sur la valence sentimentale en jeu dans la représentation d'une relation sexuelle (prise de position, règles de construction). Il apparaît en outre que cette logique de représentation (avec versus sans sentiments amoureux) module la perception du risque associé à la relation. À partir des réponses à l'indicateur "dimension sentimentale", nous avons divisé notre population en deux groupes sur la base de la moyenne ( $m=5.2)$, qui d'ailleurs correspond au milieu de l'échelle, afin de créer une variable dichotomique qui oppose les sujets selon qu'ils se déclarent plus $(N=238)$ ou moins d'accord sur cette dimension $(N=244)$. Avec cet indicateur de la valence sentimentale pris en tant que facteur, l'ANOVA réalisé met en évidence que la relation est perçue comme présentant moins de risque $(F(1,480)=21.211, p<.001)$ par les sujets se déclarant plus d'accord sur la dimension sentimentale $(m=4.9)$ comparativement à ceux qui déclarent l'être moins ( $m=6.1$; voir Figure 1$)$.

Ce résultat, conforme à notre troisième hypothèse, montre comment la perception des risques liés au sida dans le contexte d'une relation sexuelle dépend de la signification donnée à la relation: le risque perçu varie en fonction de la valence sentimentale attribuée. Ainsi, les résultats de ce dispositif quasi-expérimental corroborent de façon plausible l'hypothèse de travail issue de l'analyse des entretiens en lui conférant de la crédibilité. Ils montrent que la construction des risques implique la mobilisation des logiques représentationnelles qui donnent du sens aux relations intimes sexuelles: le sida apparaît comme une maladie associée davantage à certaines formes de sexualités dévalorisées socialement (sans sentiments amoureux).

\section{$3^{\mathrm{e}}$ étape: Opérations de triangulation et retour aux contenus comme support pour l'avancement théorique}

Les principaux résultats dégagés par l'application de la triangulation méthodologique ont confirmé la pertinence de notre hypothèse inductive sur la distinction entre relations

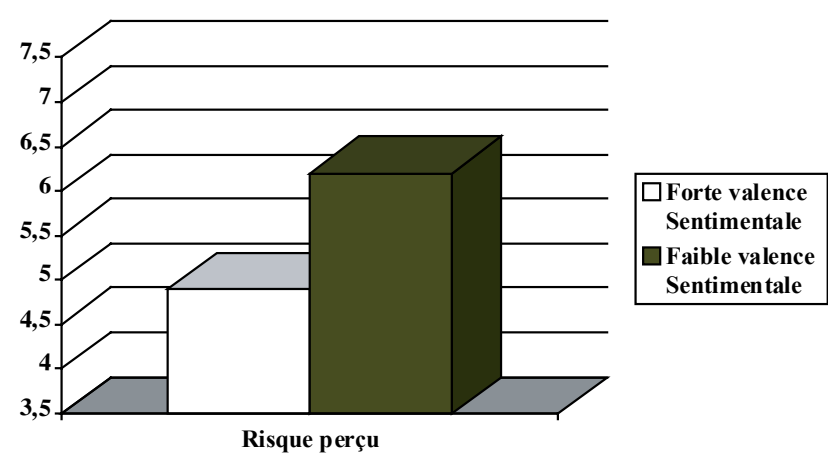

Figure 1. Perception du risque perçu par rapport au sida en fonction de la valence sentimentale attribuée. (Échelle: 1: non / 10: oui). avec ou sans sentiments amoureux. Rappelons que ces résultats corroborent pour montrer que cette construction distinctive, en tant système de représentation (contenu, organisation), induit des jeux de codification (sens, filtrage sociocognitif) et d'évaluation (préférence, idéalisation) des relations sexuelles et du risque VIH qui leur associé. A ce stade de l'analyse et de l'interprétation, l'application d'une procédure de triangulation des données (comparaison de différentes sources des données) nous a permis de confirmer la concordance de cette observation avec celles qui sont établies à partir d'autres recherches dans le domaine de la sexualité. La distinction et l'idéalisation de la sexualité par la référence à l'univers de l'amour constituent une modalité de l'assise sociale des représentations des relations sexuelles. Dans le contexte français par exemple, les résultats de l'enquête ACSF (cf. Tableau 1) montrent que plus de $90 \%$ de personnes interrogées adhérent aux opinions suivantes: "un rapport sexuel est plus satisfaisant quand on s'aime", "faire l'amour c'est faire l'un avec l'autre"' (Spencer, 1993).

L'application de cette opération comparative nous a permis d'apporter une forme supplémentaire de validité instrumentale (forme de validité obtenue par comparaison des observations à d'autres observations déjà validées; voir Rouan \& Pedinielli, 2001) par rapport à la catégorie d'analyse construite. Elle atteste de l'intérêt de considérer la sociogenèse des contenus de représentation (produits situés du point de vue socio-historique et culturel) que cette catégorie permet d'identifier et d' examiner. En effet, les constats sur la prégnance et l'importance de l'assise sociale d'une vision de la sexualité sous le prisme de l'amour peuvent suggérer que ce système de représentation renvoie à ces représentations sociales "hégémoniques" (partagée par les membres du groupe, modalité normative de coercition; Moscovici, 1988, p. 221) qui imprègnent implicitement "toutes les pratiques symboliques et affectives". Nous avons tenté de conceptualiser au regard des contributions historiques (J-L. Flandrin) et sociologiques (N. Luhmann) le caractère symptomatique des contenus représentationnels ${ }^{6}$ analysés à partir des matériaux discursifs des entretiens (Apostolidis, 2000). Cette opération visant l'application d'une triangulation interdisciplinaire nous a permis d'ébaucher une perspective d'analyse multiniveaux (sentiments intimes, construits culturels) de cette logique représentationnelle en tant que "structure sémantique" permettant d'établir un constat de correspondance entre "système" et "métasystème" (Doise, 1990).

Le rôle de cette "structure sémantique" (la distinction entre une sexualité valorisée et une sexualité dévalorisée) au niveau de la perception des risques liés au sida illustre comment l'expérience de la maladie puise dans l'univers des valeurs en jeu dans la construction sociale de la sexualité dans le contexte des sociétés occidentales. Les cultures relationnelles que met en lumière l'analyse du rôle actualisant et médiateur des représentations de l'autre dans cette construction (cognitions modalisantes sur le registre de la présupposition: pas à risque versus à risque, sincère versus

6 Elles contiennent des "informations symptomatiques" (Michelat, 1975), enracinées dans l'univers de la culture, qui attestent de la sociogenèse de leurs contenus. 
Tableau 8. Exemple d'une grille d'analyse réalisée à partir du codage sélectif du corpus (analyse horizontale).

\begin{tabular}{ll}
\hline Homme vs. femme & $\begin{array}{l}\text { L'homme a vraiment beaucoup plus besoin que la femme. elle peut s'en passe. c'est pas une obligation chez la } \\
\text { femme d'avoir ça. alors que chez l'homme. avec n'importe quelle femme c'est une nécessité. la femme attendra que } \\
\text { ça sera fait avec quelqu'un où il y a d'abord des sentiments. chez l'homme. c'est quelque chose de purement pratique } \\
\text { parce qu'il y a un plaisir sexuel égoïste. d'ailleurs ça leur plaît bien. c'est comme ça. on est comme ça. bon. c'est } \\
\text { pour ça que des fois il y a des relations bestiales. }\end{array}$ \\
\hline Propre vs. sale & $\begin{array}{l}\text { C'est pas du jour au lendemain un rapport sexuel. je trouve que c'est pas beau. il faut qu'il y ait de l'amour. sinon } \\
\text { c'est pas la peine. c'est moche. moi. je dis que ça se fait pas. c'est sale. se donner comme ça à n'importe qui. c'est } \\
\text { Beau vs. laid }\end{array}$ \\
\hline & Je crois qu'on n'a pas vraiment de souvenir du plaisir. tu vois. on a le souvenir de quelque chose de fort. il y a \\
Durable vs. éphémère & $\begin{array}{l}\text { quelque chose de fort seulement quand il y a des sentiments. le plaisir. c'est sur le moment et ce n'est pas après. ce } \\
\text { n'est pas un souvenir. ça ne reste pas quoi. }\end{array}$ \\
\hline
\end{tabular}

pas sincère, éligible versus pas éligible) s'enracinent principalement dans des conceptions et des images que sous-tend le "langage" des sentiments amoureux en tant que langage thématique (ensemble d'unités lexiques et d'argumentations qui se rattachent ou s'imprègnent d'une représentation sociale; voir Moscovici, 1961). Nous observons ici une forme de prophylaxie mettant en lumière l'intervention des schémas symboliques et intellectuels se référant à un ordre social déjà pensé, antérieur à l'avènement de la maladie considérée (Augé, 1984). Nous pouvons saisir deux types de fonctions pratiques associées à cette construction dans le contexte de peur et d'appréhension éprouvées face au sida: des fonctions expressives (idéal amoureux et ré-enchantement de l'échange intime) et des fonctions prophylactiques (sélection et évitement des partenaires, modes de construction des climats relationnels). Ces deux fonctions peuvent être illustrées à partir de deux logiques d'action analysées par rapport à l'usage du préservatif. L'une est directement en relation avec la construction amoureuse du rapport à l'autre et du climat intersubjectif du vécu sexuel (spontanéité versus préméditation, fusion versus contact). L'autre renvoie à l'efficacité perçue de ce moyen pour maîtriser le risque encouru dans le contact avec quelqu'un de potentiellement dangereux (infaillibilité).

L'application articulée de ces trois types de triangulation, du fait des recoupements opérés et des pistes d'interprétation ouvertes, nous a conduit à retourner aux contenus représentationnels afin d'approfondir l'analyse de l'état des significations en jeu dans la distinction entre relations avec ou sans sentiments amoureux. Nous avons réalisé un codage sélectif du corpus ${ }^{7}$ sur la base de cette orientation d'analyse. Cette opération nous a permis d'observer que la référence à l'univers de l'amour imprègne en tant qu'idée-source (Moscovici \& Vignaux, 1994), tant sur le plan sémantique (oppositions) qu'argumentatif (analogies, métaphores), la construction des distinctions en les inscrivant dans des univers cognitifs et sociaux plus larges et fondamentaux. Ces jeux de représentations mobilisent des systèmes d'opposition (Moscovici \& Vignaux, 1994) qui objectivent de façon

7 Opération de codage sélectif et raisonné à partir d'un objectif de recherche visant la réduction du corpus initial pour isoler et prendre en compte des unités saillantes par rapport à la problématique (Strauss et Corbin, 1990). L'analyse repose sur un travail de repérage des énoncés signifiants au sein du corpus même si leur apparition est minimale: critère de saillance. multiple la différence entre les deux formes relationnelles en tant que différence de nature, de valeur, de genre, de rapport social, ... (cf. Tableau 8).

Bien évidemment ces schémas argumentés (Moscovici \& Vignaux, 1994) ne se distribuent, ni de façon identique, ni de façon équilibrée dans les entretiens. Cependant, la mise en perspective comparée de ces schémas permet de saisir deux caractéristiques isomorphiques du régime discursif qui les sous-tend: a) l'expression d'un jugement distinctif assignant un statut d'exception aux relations sexuelles "avec" versus "sans", b) la mise en discours de ce jugement met par des thèses qui l'incarnent (par objectivation) et le légitiment (par ancrage), des thèses qui puisent dans des catégories du sens commun. Ces analyses ont permis de formuler une hypothèse de travail pour avancer dans la perspective ouverte par le concept de thêmata (Moscovici \& Vignaux, 1994; Moscovici, 2001), en examinant comment, à partir du raisonnement ordinaire, se fondent les systèmes de représentations qui régissent la vie en société. Nous avons posé l'hypothèse (Apostolidis, 2002) selon laquelle l'actualisation des différentes modes de connaissance d'autrui face aux risques liés au sida à travers des postures discursives et narratives des interviewés dévoile une forme de pensée narrative (Bruner, 2000) et exprime une vision défensive mobilisant ces catégories anthropologiquement fondamentales qui émergent dans des situations où un groupe social se sent menacé (pollution sexuelle; Jodelet, 1989). Une pensée énonçant des intentions qui puisent dans des présuppositions ancrées dans le champ de la culture, une pensée montrant des significations en action sur la réalité du monde et des relations à autrui dans le but de circonscrire le risque. L'inscription de ces logiques intentionnelles dans l'ordre des "choses" et de la vie ne donnerait-elle pas à toutes ces représentations agissantes en situation interpersonnelle leur valeur de vérité et d'efficacité, à la fois aux niveaux subjectif et intersubjectif, pour faire face à la grande peur que a suscité et suscite encore le sida en tant que maladie emblématique?

Dans la perspective que nous développions ici, les entretiens de recherche ne fournissent pas uniquement des matériaux d'appoint (exploration) mais au contraire des matériaux principaux (production des résultats). Ces matériaux constituent des supports indispensables pour l'étude des représentations (jeux d'objectivation et d'ancrage qui concourent à l'élaboration des savoirs et des significations concernant un objet) et non seulement des matériaux exploratoires fournissant des résultats à soumettre à d'autres épreuves de 
validation plus standardisées. Il est vrai que si l'entretien de recherche constitue un outil incontournable dans l'étude des contenus de représentations, il pose néanmoins plusieurs questions qu'il ne faudrait pas négliger (règles d'énonciation présidant la production discursive, fiabilité et stabilité des données, limites et biais d'interprétation au niveau de l'analyse, par exemple; Abric, 1994). La triangulation offre alors une orientation alternative de travail prometteuse et adaptée pour combiner richesse et profondeur dans l'analyse des matériaux discursifs avec rigueur et avancement contrôlé (par exemple, triangulation méthodologique: qualitatif $\rightarrow$ expérimental $\rightarrow$ qualitatif).

\section{Des Enjeux Théorico-Méthodologiques en Psychologie Sociale de la Santé.}

Pour la Psychologie Sociale de la santé, la santé et la maladie sont des lieux d'interactions complexes entre l'individuel et le collectif. L'analyse des rapports du contexte social et de la construction des risques sanitaires montre la complexité du champ des problèmes dans lesquels travaillent depuis quelques années les psychologues sociaux de la santé (par exemple, le rapport au sida met en jeu le rôle fondamental de la relation à autrui en tant que maladie représentée comme étant transmise par certaines formes de sexualité dévalorisées socialement). Dans cette contribution, nous avons cherché à poser l'intérêt d'analyser la pensée sociale profane concernant les risques sanitaires dans la perspective des représentations sociales et en appliquant une démarche de triangulation.

\section{Représentations sociales et risques sanitaires}

Le social qui intéresse la psychologie sociale de la santé, ce n'est pas tant l'environnement ou le milieu qui conditionne les comportements et provoque les stress et les maladies, c'est d'abord ce qui fournit "un système d'interprétation général du monde" (Petrillo, 2000). Le social, c'est ce qui agit à travers le caractère symbolique de la connaissance humaine pour construire et donner sens à l'expérience de maladie, objet de savoir partagé (Radley, 1994). Comme nous l'avons illustré au cours de cet article, la construction des risques sanitaires nous confronte aux facettes symboliques du raisonnement humain et interroge notre conception du sujet (sujet social versus sujet optimal de la rationalité; voir, Guimelli, 1999). En effet, à travers l'expérience de la maladie, comme à travers les domaines essentiels de la vie humaine, nous pouvons observer que "les hommes ne vivent pas seulement d'information: il leur faut aussi des significations" (Moscovici, 1992, p. 106). Le cadre théorique des représentations sociales est un support privilégié pour mettre à l'épreuve cette conception puisqu'il traite d'univers symboliques socialement partagés.

L'approche compréhensive et qualitative des représentations sociales appliquée au champ de la santé reste un moyen d'accès privilégié à l'étude des processus de formation, de diffusion et d'actualisation de la pensée sociale profane sur laquelle s'appuie l'orientation des comportements et des pratiques dans l'univers incertain de l'espace "santé-maladie". Pour la psychologie sociale de la santé, elle offre un regard paradigmatique qui consiste à mettre en place des dispositifs de recherche qui s'efforcent d'articuler différents niveaux d'interprétation afin d'échapper au réductionnisme "raisonnable" habituel aux nombreuses démarches qui répugnent à se confronter à la complexité des faits psychosociaux (Morin \& Apostolidis, 2002). Ces démarches fixent couramment le regard sur un niveau d' analyse unique, au nom d'une purification pseudo-scientifique toujours susceptible de cacher des enfermements disciplinaires (au psychologue l'intrapsychique et l'individuel; au sociologue le sociétal et le macro-social). Il est pourtant possible et souhaitable, dans le champ de la santé, de mettre à l'épreuve des méthodes d'observation et de lecture "multi-niveaux", notamment pour l'interprétation psychosociale du sens des conduites face aux risques. Dans cette perspective, il faudrait envisager le fait que les activités sociocognitives en jeu dans la construction des risques puisent dans la "boite à outils" qu' offre la culture (Bruner, 1990). Leur analyse nous impose de considérer un mode de pensée qui fonctionne sous le registre de la présupposition (c'est-à-dire, des raisonnements mettant en jeu la création d'une signification implicite qui sert de cadre pour l'interprétation; par exemple, le filtrage de l'information à partir des représentations préexistantes des sujets). Ainsi, la contextualisation de l'étude des processus sociocognitifs, en référence notamment à l'univers de la culture (Jodelet, 2002) représente un enjeu théorico-méthodologique majeur pour opérationnaliser l'articulation système/métasystème (Doise, 1990) dans l'analyse psychosociale des représentations des risques sanitaires.

\section{La triangulation: une stratégie pluri-méthodologique alternative}

Nous espérons avoir montré la pertinence et l'importance de la perspective de la triangulation, notamment pour les orientations qui s'inscrivent dans des approches qualitatives des représentations sociales. La triangulation est une stratégie alternative de recherche qui repose sur le principe d'articulation de différentes perspectives conceptuelles et méthodologiques adéquates pour étudier autant d'aspects différents que possibles du phénomène visé (Flick, 1998). Nous avons illustré ici l'intérêt de cette orientation pluri-méthodologique inductive dans la perspective des représentations sociales pour:

Étudier différents aspects de représentations et articuler une lecture multi-niveaux (élaboration des significations, construits culturels et filtrage sociocognitif de l'information, aspects symptomatiques des représentations).

Contribuer à l'avancement nécessaire dans le domaine méthodologique (articulation raisonnée des procédures ouvertes et fermées de recueil, création des dispositifs ad hoc pour étudier simultanément la double nature, produit / processus, de la représentation).

Générer de nouveaux questionnements permettant d'avancer sur le plan théorique (l'hypothèse de travail sur le thêmata).

La triangulation est une démarche complexe et contextualisée, adaptée à des objets ou des terrains spécifiques dans le domaine de la santé et de la maladie. Nous note- 
rons le potentiel de transférabilité de ces résultats pour des avancées théorico-méthodologiques dans le champ d'étude des risques sanitaires (par exemple, concernant l'élaboration des dispositifs contextualisés pour étudier dans une perspective sociocognitive la construction des risques). De plus, nous soulignerons l'intérêt de la triangulation méthodologique pour l'avancement de l'analyse (plausibilité des catégories construites par recoupement des observations) et de la conceptualisation (approfondissement, orientation). Les différentes formes qu'elle peut prendre doivent être ajustées entre elles et justifiées sur le plan conceptuel. Plus globalement, elle offre un cadre pour considérer la pratique de la pluri-méthodologie en psychologie sociale de la santé qui permet:

- D'apporter crédit, richesse et consistance aux analyses par la complémentarité des différentes méthodes de recueil en combinant leurs avantages et en réduisant leurs limites.

De stimuler la créativité méthodologique et l'avancement sur la formalisation d'une critériologie de validation qui ne définit pas seulement l'objet à partir des conditions de la méthode (critères pour penser l'éclectisme et la flexibilité).

De disposer d'une approche heuristique pour dépasser les oppositions stériles et tout à fait inappropriées entre méthodes qualitatives et quantitatives ou expérimentales (continuum qualitatif/quantitatif/expérimental).

De produire des connaissances transférables et cumulatives pour le développement méthodologique, notamment concernant la construction des dispositifs expérimentaux basés sur des "variables indépendantes molaires" (Deconchy, 2002) pour étudier la dynamique sociocognitive des représentations sociales.

Bien évidemment, loin d'épuiser les questionnements soulevés, les propositions avancées ici invitent à de futures opérationnalisations pour approfondir et définir de façon plus extensive les conditions d'application des différentes formes de triangulation. En psychologie sociale de la santé, la perspective de la triangulation dépasse les oppositions classiques entre paradigmes méthodologiques et tend vers une complémentarité des méthodes ayant comme critère de choix l'adaptation des outils aux objectifs et à l'avancement de la problématique de la recherche concernant l'étude de la pensée sociale dans le domaine de la santé et de la maladie.

\section{Références}

Abric, J.-C. (1987). Coopération, Compétition et représentations sociales. Cousset: DelVal.

Abric, J-C. (1994). Méthodologie de recueil des représentations sociales. Em J-C. Abric (Org.), Pratiques sociales, représentations (pp. 59-82). Paris: PUF-Psychologie Sociale.
Apostolidis, T. (1994). Représentations sociales de la sexualité et du lien affectif: la logique relationnelle des comportements sexuels et la prévention du sida. Em M. Calvez, G. Paicheler, Y. Souteyrand (Orgs), Connaissances, représentations, comportements: Sciences sociales et prévention du SIDA (pp. 77-85). Paris: Documents de 1'ANRS/Coll. Sciences Sociales et sida.

Apostolidis, T. (2000). Le rapport au sexuel et la "sémiotique" de l'amour: marquage socioculturel et climats relationnels. Journal des anthropologues, 82-83, 339-356.

Apostolidis, T. (2002). Représentations d'autrui dans le contexte d'une relation intime: remarques topologiques sur les croyances. Psychologie et société, 5, 13-41.

Apostolidis, T. (2003). Représentations sociales et triangulation: enjeux théorico-méthodologiques. Em J-C. Abric (Org.), Méthodes d'étude des représentations sociales (pp 13-35). Saint-Agne: Erès.

Augé, M. (1984). Ordre biologique, ordre social: la maladie forme élémentaire de l'événement. Em M. Augé \& C. Herzlich (Orgs.), Le sens du mal. Anthropologie, Histoire, Sociologie de la Maladie (pp. 35-91). Paris: Éditions des archives contemporaines.

Bardin, L. (1977). L'analyse de contenu. Paris: PUF

Beauvois, J-L. \& Deschamps, J-C. (1990). Vers la cognition sociale. Em R. Ghiglione, C. Bonnet \& J-F. Richard (Orgs), Traité de psychologie cognitive 3 (pp 1-110). Paris : Dunod.

Bruner, J. (1990). ...car la culture donne forme à l'esprit. Paris: ESHEL.

Bruner, J. (2000). Culture et modes de pensée. Paris: Retz.

Deconchy, J.-P., De Koning, M. \& Medioni, F. (1993). Croyances, filtres cogitifs et représentations anthropologiques dominantes. Archives de Sciences sociales des religions, 82, 183-204.

Deconchy, J.-P. (2002). Représentations et processus idéologiques: effets d'enveloppe et expérimentation. Nouvelle Revue de Psychologie Sociale, 1, 90-98.

Demazière, D. \& Dubar, C. (1997). Analyser les entretiens biographiques. Paris: Nathan.

Denzin, N. (1978). The research act. Chicago: Aldine.

Denzin, N. \& Lincoln, Y. (1998). Emerging the field of qualitative research. Em N. Denzin \& Y. Lincoln (Orgs.), Strategies of qualitative inquiry (pp. 1-34). London: Sage.

Di Giacomo J.-P. (1981). Aspects méthodologiques de l'analyse des représentations sociales. Cahiers de psychologie cognitive, 1, 397-422.

Deschamps, J. C. \& Clémence, A. (1987). L'explication quotidienne. Cousset : Delval.

Doise, W. (1990). Les représentations sociales. Em R. Ghiglione, C. Bonnet, J-F. Richard (Orgs.), Traité de psychologie cognitive 3 (pp. 111-174). Paris: Dunod.

Doise, W., Clemence, A. \& Lorenzi-Cioldi, F. (1992). Représentations sociales et analyses de données. Grenoble: PUG.

Echebarria Echabe, A. \& Paez Rovira, D. (1989). Social representations and memory: the case of AIDS. European Journal of Social Psychology, 19, 543-551.

Farr, R. \& Moscovici, S. (Orgs.) (1984). Social representations. Cambridge: Cambridge University Press.

Flament, C. (1984). From the bias of structural balance to the representation of the group. Em R. Farr, S. Moscovici (Orgs.), Social representations (269-285). Cambridge: Cambridge University Press. 


\section{T. Apostolidis}

Ficher, G. N. \& Tarquinio, C. (1999). Les aspects psychosociaux dans les méthodologies en psychologie de la santé. Pratiques Psychologiques, 4, 31-43.

Flick, U. (Org) (1991). La perception quotidienne de la santé et de la maladie. Théories subjectives et représentations sociales. Paris: L'Harmattan.

Flick, U. (1992). Triangulation Revisited: Strategy of Validation or Alternative?. Journal of the Theory of Social Behavior, 22(2), 176-197.

Flick, U. (1998). An introduction to qualitative research. London: Sage.

Guimelli, C. (1999). La pensée sociale. Paris: PUF-Collection Que sais-je..

Heider, F. (1958). The psychology of interpersonal relations. New York: Wiley.

Herzlich, C. (1969). Santé et maladie: analyse d'une représentation sociale. Paris: Mouton.

Herzlich, C. (2001). Les représentations sociales de la santé et la santé en mutation: un regard rétrospectif et prospectif sur la fécondité d'un concept. Em F. Buschini \& N. Kalampalikis (Orgs.), Penser la vie, le social, la nature. Mélanges en l'honneur de S. Moscovici (pp. 189-200). Paris: Editions de la MSH.

Hewstone, M. (1986). Understanding attitudes to the European Community: A Social-psychological study in four member states. Cambridge: Cambridge University Press.

Hewstone, M. (1989). Représentations sociales et causalité. Em D. Jodelet (Org.), Les Représentations Sociales (pp. 252-274). Paris: PUF.

Hewstone, H. \& Augoustinos, M. (1998). Social attributions and social representations.Em U. Flick (Org.), Psychology of the social (pp. 60-76). Cambridge: Cambridge University Press.

Janesick, V. (1998). Stretching Exercises for Qualitative Researchers. London: Sage.

Jodelet, D. (1984). Réflexions sur le traitement de la notion de représentation sociale en psychologie sociale. Communication - Information, 6(2-3), 15-42.

Jodelet, D. (1989). Folies et représentations sociales. Paris: PUF.

Jodelet, D. (1991). Représentation sociale. Em Grand dictionnaire de la psychologie (pp. 668-672). Paris: Larousse.

Jodelet, D. (1992). Les représentations sociales. Le Courrier du CNRS, 79, 109.

Jodelet, D. (2002). Les représentations sociales dans le champ de la culture. Information sur les Sciences Sociales, 41(1), 111-133.

Jodelet, D. (2003). Aperçus sur les méthodologies qualitatives. Em S. Moscovici \& F. Buschini (Orgs.). Les méthodes des sciences humaines (pp 139-162). Paris: PUF.

Joffe, H. (1999). Représentations sociales et psychologie de la santé. Pratiques Psychologiques, 4, 15-30.

Joffe, H. (2003). Risk: from perception to social representation. British Journal of Social Psychology, 42, 55-73.

Maisonneuve, J. (1966). Psycho-sociologie des affinités. Paris: PUF.

Michelat, G. (1975). Sur l'utilisation de l'entretien non directif en sociologie. Revue Française de Sociologie, XVI, 229-247.
Michelat, G. \& Simon, M. (1977). Classes, religion et comportement politique. Paris: Fondation des Sciences politiques.

Morin, M. (1996). Perspectives de recherches pour l'étude empirique de l'explication sociale des maladies. Psychologie Française, 41-42, 147-154.

Morin, M. \& Apostolidis, T. (2002). Contexte social et santé. Em G. Fischer (Org.), Traité de psychologie de la santé (pp. 463489). Paris: Dunod.

Moscovici, S. (1961). La psychanalyse, son image et son public. Paris: PUF.

Moscovici, S. (1984). Introduction: le domaine de la psychologie sociale. Em S. Moscovici (Org.). Psychologie Sociale (pp. 522). Paris: PUF.

Moscovici, S. (1988). Notes Towards a Description of Social Representations. European Journal of Social Psychology, 18(3), 211-250.

Moscovici, S. (2001). Why a Theory of Social Representations. Em K. Deaux \& G. Philogene (Orgs.), Representations of the social: Bridging Theoretical Traditions (pp 18-61). Oxford: Blackwell.

Moscovici, S. \& Vignaux, G. (1994). Le concept de thêmata. Em C. Guimelli (Org.), Structures et transformations des représentations sociales (pp. 26-72). Paris: Delachaux et Niestlé.

Paicheler, H. (1984). L'épistémologie du sens commun. Em S. Moscovici (Org.), Psychologie sociale (pp. 277-307), Paris: PUF.

Petrillo, G. (2000). La psychologie sociale dans le cadre des sciences sociales de la santé: question et prospectives. Em Petrillo, G. (Org.), Santé et société (pp. 13-44). Lausanne: Delachaux et Niestlé.

Radley, A. (1994). Making sense of illness. London: Sage.

Rouan, G. \& Pedinielli, J-L (2001). Aspects épistémologiques des méthodes qualitatives. Em M. Santiago-Delfosse M. \& G. Rouan (Orgs.), Les méthodes qualitatives en psychologie (pp. 39-59). Paris: Dunod.

Smith, J. (1995). Semi-structured interviewing and qualitative analysis. Em J. Smith, R. Harré \& L. Van Langenhove (Orgs.), Rethinking methods in psychology (pp. 9-26). London: Sage.

Spencer, B. (1993). Contexte normatif du comportement sexuel et choix des stratégies de prévention. Population, 5, 1411-1436.

Spira, A., Bajos, N. \& le Groupe ACSF. (1993). Les comportements sexuels en France, Rapport au ministère de la Recherche et de l'Espace. Paris: Éditions La documentation Française.

Strauss, A. (1989). Miroirs et masques: une introduction à l'interactionnisme. Paris: Éditions Métailé.

Strauss, A.L. \& Corbin, J. (1990). Basics of qualitative research: Grounded theory procedures and techniques. California: Sage.

Willig, C. (2001). Introducing qualitative research in psychology. Buckingham: Open University Press.

Recebido em 17.01.2006

Primeira decisão editorial em 19.04.2006

Versão final em 15.05.2006

Aceito em 10.06.2006 